\title{
Bijective model of time and J.T. Fraser's Nowless Universe
}

Amrit S. Šorli

Bijective Physics Institute, Slovenia

sorli.bijective.physics@gmail.com

\begin{abstract}
In bijective modelling, the physical reality is represented by the set $\mathrm{X}$, the model of physical reality by the set $\mathrm{Y}$. Every element in the set $\mathrm{X}$ has exactly one correspondent element in the set $\mathrm{Y}$. Set $\mathrm{X}$ and set $\mathrm{X}$ are related by the bijective function $f: X \rightarrow Y$. Bijective modelling is confirming that time is the duration of given system entropy increasing in time-invariant space. Time-invariant space is the fundamental arena of the Nowless Universe.
\end{abstract}

Keywords: Space; Time; Entropy; Nowless Universe; Bijective modelling.

\section{Introduction}

Bijective research methodology sees time as a duration of a given system entropy increase that happens in space. Out of this follows that we have in the bijective model of entropy in the set $\mathrm{X}$, that represents physical reality, three elements: space $S_{X}$, time $\Delta t_{X}$, entropy increase $\Delta S_{X}$ as shown in formula below. The duration of entropy increase $\Delta S$ is the elapsed time $\Delta t$ :

$$
X:\left\{S_{X}, \Delta t_{X}, \Delta S_{X}\right\}
$$

In the set $\mathrm{Y}$ we have the following correspondent elements:

$$
Y:\left\{S_{Y}, \Delta t_{Y}, \Delta S_{Y}\right\}
$$

Bijective modelling confirms that time is a duration of entropy increase of a given physical system happening in space.

\section{Bijective model of time, space and entropy}

Here the crucial question will be solved: "How the time, that is duration of entropy increasing, could be the $4^{\text {th }}$ dimension of space-time? The bijective analysis of Special Relativity formalism for $4^{\text {th }}$ dimension in its original form shows that time $t$ is not $X_{4}$ :

$$
X_{4}=i c t
$$


Out of Eq. (3) it follows [1]:

$$
X_{4} \neq t
$$

The forth coordinate of Minkowski manifold can be called "temporal" only in the sense that time as duration is its element, but time itself is not the $4^{\text {th }}$ dimension of spacetime. $X_{4}$ is spatial in the same way as dimensions $X_{1}, X_{2}, X_{3}$. Time, as a duration, is a physical property of every material change, i.e. motion happening in space, including the increase of entropy.

We have experimental data and theoretical models only related to the duration of material change, i.e. motion has some physical impact on space or is related to space in some other relation. The only real relation between space and time is that time is the duration of the change in space, which means that space is time-invariant. Increasing of entropy $\Delta S$ of a given physical process happens in time-invariant space, where time is the duration of the entropy increase.

The product of time $t$, as duration, with the light speed $c$ gives the spatial distance $d=c t$. Einstein has added to this spatial distance the imaginary number $i$, so we write $X_{4}=i d$. This imaginary distance in the Minkowski model has no bijective correspondence with the physical world; the relation $f: i d_{X} \rightarrow i d_{Y}$ is not true, the imaginary distance $i d$ is not existing in the physical world.

To solve this puzzle in Special Relativity, the space-time interval $S$ has been defined as: $S^{2}=$ $c^{2} t^{2}-\left(X^{2}+Y^{2}+Z^{2}\right)$. With the introduction of natural units: $c=\hbar=1$, the space-time interval becomes: $S^{2}=t^{2}-\left(X^{2}+Y^{2}+Z^{2}\right)$ and the fourth coordinate of Minkowski manifold was interpreted as time $t$; in its original form $X_{4}=i c t$ it turned into $X_{4}=t$. Time has been fully merged with space and still today we think that time is the $4^{\text {th }}$ dimension of space-time.

In the time-invariant space we do not have time-arrow. Time is not pointing anywhere, because it is the duration of material change running in the time-invariant space. Time-arrow as the element of the model of the physical reality has no correspondent model in physical reality. We have several models of time-arrows, but none has correspondent time-arrow in physical reality: thermodynamic time-arrow [2] does not exist in physical reality because entropy is increasing in time-invariant space. Also, cosmological time-arrow [3] has no physical existence because universe is developing in time-invariant space. 
In this article we show that there is no flow of time in physical reality. Material changes, i.e. motion, run in time-invariant space and time is their duration. Recent research is also suspicious about the flow of some hypothetical physical time: "Third, the statement that the flow of time causes entropy to increase and not the other way around is doubly misleading. Entropy, by itself, cannot be said to increase or decrease. The reason is that entropy is a state function, i.e., it is defined for a well-defined system at equilibrium. As such, it is not a function of time. The flow of time is not the cause of entropy increase! The "flow of time" (if it flows at all) has nothing to do with entropy increase!" [4].

\section{Time-invariant space is confirming time translation symmetry (TTS) and denying existence of time-reversibility}

The model of time-invariant space is confirming the validity of time translation symmetry (TTS) [5], which is a rigorous way to formulate the idea that the laws of physics are the same throughout history. The history is running in the time-invariant space where the laws of physics are invariant on time and time is merely the duration of material changes.

In the time-invariant space there is no past and no future. Material changes are irreversible and do not run in some physical time. That's why the time-symmetry model, where the physical laws are symmetric in time, has no bijective correspondence in the physical world. No physical process can be reversed in some physical time, because time is just duration of the given physical process.

In bijective modelling the duration is a discrete quantity. Every elapsed time is the sum of Planck times [6]:

$$
t=t_{P 1}+t_{P 2}+\cdots+t_{P n}=\sum_{i=1}^{n} t_{P i}
$$

Also other research is confirming that time may be seen as concrete quantity: "Time may be considered as a discrete quantity" [7]. Planck time $t_{P}$ has physical existence but is not part of space, it is part of duration. In this virtue, Planck time is the fundamental unit for measuring the duration of the increasing of entropy that runs in a time-invariant space. The model of time reversal symmetry (T-symmetry) has no bijective correspondence in the physical world. The equation below has no physical meaning:

$$
T: t \rightarrow-t \quad(6)
$$


Experimental research is confirming that time reversal symmetry is a model that has no bijective correspondence with the physical world [8].

The idea of Stueckelberg and Dirac that particles could move back in time becoming antiparticles was never experimentally proved: "This configuration can be understood as pair annihilation; it was already known at that time that a particle running backwards in time can be understood, and observed, as an antiparticle going forward in time. This phenomenon occurs in the solutions of the Dirac equation, where the wave function of a particle going backward in time, under charge conjugation, describes an antiparticle moving forward in time. Dirac, in this way, discovered the positron, the antiparticle of the electron. Stueckelberg therefore called this configuration pair annihilation in classical mechanics" [9].

The positron discovery does not prove that it is the electron moving backward in time. Experimental data prove that they are both discovered and they both move and exist in the same universal space. If positron would really exist in some negative time, the measurement system also should be there in the negative time that in Minkowski manifold is represented by the past light cone (Figure 1):

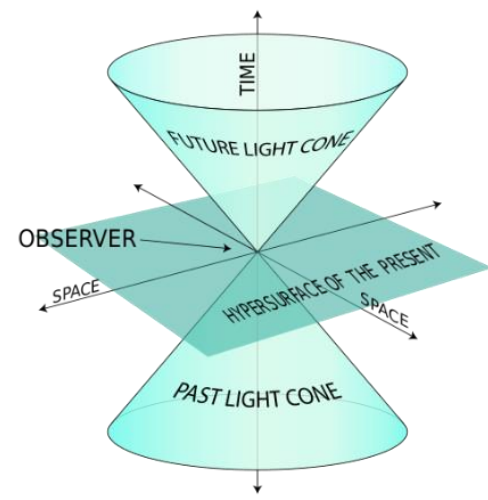

Figure 1: Minkowski manifold with positive and negative time

The universal space, in which electron and positron are discovered, is time-invariant in the sense that time is not the $4^{\text {th }}$ dimension of space-time and that this $4^{\text {th }}$ dimension has neither positive nor negative sign. The picture of Minkowski manifold has no bijective correspondence with the physical world.

Recent research suggests that two different types of time are existing in the universe: "We are now in a position to discuss the properties of entropy flow associated with the relativistic Boltzmann Equation. This result, that there are intrinsically two types of time, 
the observable time $t$ associated with the Maxwell equations and Einstein's formulation of special relativity (appearing in the Lorentz transformation), and the underlying universal parameter $\tau$ of dynamical evolution, as originally conceived by Newton, is a consequence of the Stueckelberg-Horwitz-Piron formulation of relativistic mechanics" [9].

The so called "invariant universal world time" $\tau[10]$ has been never observed and measured, and has no bijective correspondence in the physical reality. We suggest that the only existing time is the observable time $t$, which is the duration of material change, i.e. motion in the timeinvariant universal space.

In today's standard quantum mechanics (SQM), we are still having the wrong imagination that quantum objects exist in some physical time: "It is a fundamental question in standard quantum mechanics (SQM) of what type of restrictions the Schrödinger evolutionism poses on the behavior in time of basic objects. In particular, it is of interest to ask if SQM allows for selfadjoint operators having the so-called Lyapunov property, that is, monotonicity of the expectation value irrespective of the initial state of the system. Clearly, such an operator would indicate the direction of time" [11]. Experimental physics is confirming that basic objects are existing only in space, and time is the duration of their motion. We suggest that the duration cannot have a direction, the existence of the arrow of time as direction of time would be then excluded.

\section{Bijective modelling of relation between EPR-type entanglement and entropy}

In the bijective model, the increase of entropy $\Delta S$ requires the duration $\Delta t$ :

$$
\Delta S \rightarrow \Delta t
$$

EPR-type entanglement is immediate, it is carried by the time-invariant space having its physical origin in the superfluid quantum vacuum [12]. The elapsed time of EPR-type entanglement is zero, which means that EPR-type entanglement has no entropy:

$$
\Delta t=0 \rightarrow \Delta S=0
$$

Several researches suggest that entanglement and entropy are related: "The entanglement can be quantified by an entropy. One can define it as the measure of the information about quantum states which is lost when these states cannot be observed. In many-body systems, which are 
the subject of the present work, "observable" and "unobservable" states can be located in different regions" [13].

We suggest that by EPR-type entanglement there is no loss of information, which can only take place when information transfer has duration; no duration means no information loss. Entanglement and entropy are not related. In this view also Misra, Prigogine and Courbage's no-go theorem that excludes possibility of immediate information transfer [11] seems questionable, because the time-invariant space can be seen as the immediate medium of entanglement $[1,12]$.

\section{The velocity of clocks and entropy depends on the variable density of time-invariant space}

As example, let's imagine to have two identical systems, two boxes with two chambers: the gas is in the left chamber, vacuum is in the right chamber. One box is on the Earth's surface, the other is in the interstellar space. When we open the holes in the walls between chambers, the gas will be distributed uniformly in both chambers. The entropy of both systems will equally increase.

The time of the entropy increase $\Delta S_{E}$ in the system on the Earth will be $\Delta t_{E}$. Let's take that $\Delta t_{E}$ is one second. The time $\Delta t_{I S}$ of the entropy increase $\Delta S_{I S}$ of the system in the interstellar space far away from stellar objects (theoretically infinitely distant from the Earth) will be longer for 0.000000000695915 of a second [12]:

$$
\begin{gathered}
\Delta S_{E}=\Delta S_{I S} \\
\Delta t_{E}=\Delta t_{I S}-6.96 \cdot 10^{-10} S
\end{gathered}
$$

This is because material changes, rate of clocks included, run faster in the interstellar space where the density of superfluid quantum vacuum, that is the physical origin of time-invariant universal space, has maximum density $\rho_{\max }$. The density of superfluid vacuum $\rho_{\min }$ on the Earth's surface is as follows:

$$
\rho_{\text {min }}=\rho_{\text {max }}-\frac{3 m}{4 \pi \cdot(r+d)^{3}}
$$

where $m$ is the mass of the stellar object, $r$ is the radius of the stellar object ad $d$ is the distance from the centre of the stellar object [12]. The variable density of superfluid quantum 
vacuum defines the velocity of the entropy increase of a given system and the velocity of material changes in general.

\section{The model of time-invariant universal space has strong epistemological correspondence with J.T. Fraser's Nowless Universe}

J.T. Fraser said in his article "Human Temporality in a Nowless Universe": The human experience of time is that of an ever-changing present in which the future becomes past. By sharp contrast, the laws that govern the physical world do not allow any meaning to be assigned to a present. Since future and past, and the metaphor of time's flow make sense only with reference to a now, physical processes cannot, and do not, provide for a preferential direction of change. This paper shows how the undirected time of the physical world and the directed temporalities of life and of conscious experience may nevertheless be accommodated within a natural philosophy of time" [14]. The ever-changing present is the ever-changing flow of material change that runs in the time-invariant universal space. Humans, we experience timeinvariant universal space as now.

J.T. Fraser said in the same article: "In the human experience of time, idea about future and past are combined with interpretations of current sense impressions. The ceaseless change in the complement of events assigned, respectively, to time future, time past and time present is called the flow of time. Curiously, neither the idea of future, past and present nor the metaphor of time's directed flow can be given any meaning for the physical world as it is seen through physics. This paper suggests a way in which human temporality may, nevertheless, arise from the nowless, non-flowing-undirected-time of the physical universe" [14]. Yes, the ceaseless change that runs in universal space is not proving the existence of some physical time flow in the universe. Yes, human temporality arises from the observation of ceaseless change running in time-invariant universal space where time exists in its nowless, non-flowing, undirected form. This nowless time we name a fundamental time that is the numerical sequential order of material changes, i.e. motion running in time-invariant space. Once the fundamental time is measured from the side of the observer it turns into emergent time that is duration. There is no duration without the measurement from the side of the observer [6].

Alfred North Whitehead has denied the reality of instant and introduced the concept of time by that of "process" [15]. We have shown in this article that all things in the universe are instantly related (entangled) via time-invariant space. Material change, i.e. motion is a "process of change" where time is merely the numerical sequential order of events. Events do not run in time, they run in nowless universe. In this perspective, J.T. Fraser's view on time is more 
adequate than Whitehead's, namely, material changes, i.e. motion, do not have a duration of their own; they only have their own velocity. The duration, i.e. the elapsed time, enters in existence when time as numerical order of change is measured by an observer. The fundamental unit of numerical order of changes is the Planck time; every elapsed time $t$ is a sum of Planck times, see equation (5). This has immense implications on cosmology and on science in general. Our temporal view on the universe running in some linear physical time should be replaced with the view where the universe runs in the time-invariant space that we humans experience as the present moment. This ever-changing present moment is the only one that exists. All rest is just human imagination: there is no physical past and there is no physical future. The irreversible flow of changes runs in time-invariant universal space.

\section{Conclusions}

Progress of physics is in building the most accurate models of the physical world. A bijective research methodology is a very useful tool in this task; it confirms that entropy increases in a time-invariant space and that time is the duration of entropy increase. The velocity of entropy increase depends on the variable energy density of superfluid quantum vacuum, that is the physical origin of the time-invariant universal space that is the fundamental arena of Fraser's Nowless Universe.

\section{References:}

[1] Fiscaletti, D., Sorli, A. Searching for an adequate relation between time and entanglement. Quantum Stud.: Math. Found. 4(4), 357-374 (2017). https://link.springer.com/article/10.1007/s40509-017-0110-5.

[2] 't Hooft, G. Time, the Arrow of Time, and Quantum Mechanics. Front. Phys. 6, 81 (2018). https://doi.org/10.3389/fphy.2018.00081.

[3] Hawking, S.W. Arrow of time in cosmology. Phys. Rev. D 32, 2489 (1985). https://doi.org/10.1103/PhysRevD.32.2489.

[4] Ben-Naim, A. Entropy and Time, Entropy 22(4), 430

(2020). https://doi.org/10.3390/e22040430.

[5] Lehto, M., Nielsen, H.B., Ninomiya, M. Time translational symmetry, Phys. Lett. B 219(1), 87-91 (1989). https://doi.org/10.1016/0370-2693(89)90843-5.

[6] Fiscaletti, D., Sorli, A.S. Perspectives of the numerical order of material changes in timeless approaches in physics. Found. Phys. 45(2), 105-33 (2015).

https://doi.org/10.1007/s10701-014-9840-y. 
[7] Lucia, U., Grisolia, G., Kuzemsky, A.R. Time, Irreversibility and Entropy Production in Nonequilibrium Systems, Entropy 22(8), 887 (2020). https://doi.org/10.3390/e22080887.

[8] Müller, C., Guan, S., Vogt, N., Cole, J.H., and Stace, T.M. Passive On-Chip

Superconducting Circulator Using a Ring of Tunnel Junctions. Phys. Rev. Lett. 120, 213602 (2018). https://doi.org/10.1103/PhysRevLett.120.213602, https://arxiv.org/abs/1709.09826.

[9] Horwitz, L.P. The Relativistic Boltzmann Equation and Two Times, Entropy 22(8), 804 (2020). https://doi.org/10.3390/e22080804.

[10] Horwitz, L.P. Stuckenberg-Horwitz-Piron Canonical Quantum Theory in General Relativity and Bekenstein-Sanders Gauge Fields for the TeVeS (2019). doi:

10.5772/intechopen.88154.

[11] Strauss, Y., Silman, J., Machness, S., Horwitz, L.P. Study of a self-adjoint operator indicating the direction of time within standard quantum mechanics. Comptes Rendus Mathematique 349(19-20), 1117-1122 (2011). https://doi.org/10.1016/j.crma.2011.09.007.

[12] Šorli, A.S. Mass-Energy Equivalence Extension onto a Superfluid Quantum Vacuum. Sci Rep 9, 11737 (2019). https://doi.org/10.1038/s41598-019-48018-2.

[13] Fursaev, D.V. Entanglement Entropy in Critical Phenomena and Analogue Models of Quantum Gravity. Phys. Rev. D 73, 124025 (2006). https://doi.org/10.1103/PhysRevD.73.124025.

[14] Fraser JT. Human Temporality in a Nowless Universe. Time \& Society. 1992;1(2):159173. doi:10.1177/0961463X92001002002.

[15] Rémy Lestienne. In Support of Whitehead's Time, KronoScope, Volume 18: Issue 1, (2018), https://doi.org/10.1163/15685241-12341400. 